\title{
The European Materials Research Society (EMRS) Spring Meeting 2014 Symposium I
}

The European Materials Research Society (EMRS) Spring Meeting 2014 Symposium I-Solution Processing and Properties of Functional Oxide Thin Films and Nanostructures was organized to provide an overview of the current state of research on the synthesis and processing of solution-derived oxide and nanocomposite thin films and nanostructures, including their properties and applications, and offered a forum for the interchange of information and expertise.

The EMRS Spring Meeting was held on 26-30 May 2014 in the Congress Center in Lille, France. The symposium on Solution Processing attracted a large number of participants, showing the great interest among materials scientists inside and outside Europe in a dedicated platform on solution processing of inorganic (nano) materials. There was a high involvement of young researchers, in particular graduate students in the symposium. In total, 257 accepted abstracts were presented in the symposium. There were 26 invited and 65 contributed oral presentations distributed over 17 oral sessions, and 166 posters in 3 evening poster sessions.

The symposium addressed all aspects of solution processing of oxide materials with specific functionalities, ranging from materials synthesis, low temperature processing, microstructural and functional characterization, nanoparticles, colloidal dispersions, nanowires, nanosheets, thin films, hybrid materials, printing and patterning of materials, to issues of large scale processing and scale-up. Special sessions were dedicated to electronic, optoelectronic, ferroelectric, photovoltaic and other types of electronic and energy materials. One of the sessions entitled "Japan in Motion" was focused entirely on some of the current materials research activities in the open research centers of the World Premier International Research Center Initiative (WPI) in Japan. Two graduate students received an award for their scientific achievements. Also three poster prizes were given to excellent graduate students.

We would like to thank all contributors to the symposium, the session chair-persons, symposium assistants and our sponsors Umicore and FOM Technologies. We also thank the Advisory Board for their advice. We would like to thank the European Materials Research Society for their support and organization of the meeting.

The symposium organizers,

Johan E. ten Elshof, University of Twente, Netherlands, email j.e.tenelshof@utwente.nl.

Narcís Mestres, Institute of Materials Science Barcelona, Spain

An Hardy, University of Hasselt, Belgium

Barbara Malič, Jožef Stefan Institute, Slovenia

Geoffrey L. Brennecka, Colorado School of Mines, CO, USA 